\title{
DEL LÉXICO A LA GRAMÁTICA: RUTAS DE GRAMATICALIZACIÓN EN MAKÁ
}

\author{
From lexicon to grammar: grammaticalization paths in maká
}

Temis Lucía Tacconi*

\begin{abstract}
RESUMEN
En este artículo abordamos diversas rutas de gramaticalización en una lengua de la familia mataguaya, el maká. A partir de los datos recolectados en terreno y mediante el método de la reconstrucción histórica, proponemos cuatro instancias de morfemas con funciones gramaticales que provienen ya sea de lexemas nominales, como es el caso de la palabra 'hijo' que deviene sufijo diminutivo y afectivo o el de la palabra it'ij 'decir' que conforma una construcción causativa analítica; de morfemas gramaticales que se vuelven más gramaticales, como es el caso del sufijo desiderativo que toma valor aspectual; o de los aplicativos que parecen provenir de construcciones de verbos seriales.

Partimos de la premisa de que es posible analizar diversas instancias de gramaticalización aun en lenguas con escaso registro histórico dado que la multifuncionalidad de los morfemas de la lengua se constituye como un indicio sincrónico de los cambios diacrónicos de la lengua.
\end{abstract}

Palabras clave: gramaticalización, familia mataguaya, morfología

\begin{abstract}
In this article, we address various routes of grammaticalization in a language of the Mataguayan family, Maká. From the data collected in the field and through the method of historical reconstruction, we propose four instances of morphemes with grammatical functions that come either from nominal lexemes, as is the case of the word 'son' that becomes a diminutive and affective suffix or the one of the word it'ij 'to say' that forms an analytical causative construction; either of grammatical morphemes that become more grammatical, as is the case of the desiderative suffix that takes aspectual value or of the applicatives that seem to come from constructions of serial verbs.

We start from the premise that it is possible to analyze various instances of grammaticalization even in languages with scarce historical record since the multifunctionality of the morphemes of the language is constituted as a synchronic indication of the diachronic changes of the language.
\end{abstract}

Key Words: grammaticalization, Mataguayan family, morphology

*Universidad de Buenos Aires - CONICET. Dra. en Lingüística. Argentina.

La investigación cuyos resultados se vuelcan en este artículo ha sido posible gracias a una beca postdoctoral otorgada por el Consejo Nacional de Investigaciones Científicas y Técnicas de Argentina y al financiamiento UBACyT 2016-2019 20020160100106BA.

Correo electrónico: temis.tacconi@gmail.com

Recepción: 18-01-2018 Aceptación:09-02-2018 


\section{Introducción}

En un estudio sincrónico de las lenguas podemos encontrar diversos elementos que dan cuenta de procesos históricos de cambio del léxico a la gramática, en otras palabras, de procesos de gramaticalización. Desde una perspectiva descriptiva tipológico-funcional, este trabajo tiene por objetivo examinar algunas rutas de gramaticalización en maká (familia mataguaya) que ocurren en el dominio de los sufijos diminutivo y aspectual, así como en las construcciones aplicativas y causativas.

Se denomina gramaticalización al proceso por el cual las formas léxicas y las construcciones sintácticas bajo ciertas condiciones y en ciertos contextos pasan a tener funciones gramaticales y una vez gramaticalizadas continúan desarrollando nuevas funciones (Hopper y Traugott, 1993, p. 15). Este fenómeno puede abordarse tanto desde una perspectiva sincrónica como desde una diacrónica (Lehmann, 2004, p. 2). En este artículo nos interesa, por un lado, referirnos a la cuestión de cómo estudiar estos fenómenos en lenguas que no poseen una documentación de larga data, como es el caso de las lenguas indígenas; y, por otro lado, explicar la coexistencia de dos o más funciones para una misma forma en la lengua maká.

En el caso del maká, los procesos de gramaticalización involucran diversos lexemas independientes que sincrónicamente funcionan también como afijos gramaticales. Este es el caso de los aplicativos que provienen de verbos de posición, así como del sufijo diminutivo que tiene su origen en el término -as 'hijo'. Asimismo, el sufijo desiderativo -hiyu se gramaticaliza como sufijo de aspecto ingresivo en determinados contextos. Finalmente, existe en maká una construcción causativa con el verbo 'decir' que se encuentra en proceso de gramaticalización. Es decir que en este artículo abordaremos diversos fenómenos que abarcan las distintas instancias de la gramaticalización: procesos que involucran lexemas que toman funciones gramaticales (2.1 y 2.2), morfemas gramaticales que toman más funciones gramaticales (2.3), y construcciones sintácticas que al final del proceso conforman una unidad compacta (2.4).

\subsection{La lengua maká: características sociolingüísticas y rasgos gramaticales sobresalientes}

El maká pertenece a la familia mataguaya, al igual que el wichí, el chorote y el nivaclé. De acuerdo con el Censo Nacional para Pueblos Indígenas (DGCEE, 2014), es la lengua hablada por aproximadamente 1900 personas en diversos distritos de la República del Paraguay: Mariano Roque Alonso, Falcón e Ita Paso. Si bien el español tiene una mayor difusión que hace unos años, la lengua de comunicación preferida dentro de la comunidad es todavía el maká. Las mujeres mayores y los niños en edad preescolar son monolingües en la lengua vernácula. Estos últimos adquieren el español y el guaraní cuando ingresan a la escuela. 
Con respecto a los rasgos gramaticales sobresalientes, el maká es una lengua aglutinante con tendencia a la polisíntesis (Gerzenstein, 1995). Presenta marcación en el núcleo y distinción entre nombres alienables e inalienables, los segundos llevan un marcador posesivo obligatorio. Entre los nombres inalienables se encuentran los términos de parentesco y las partes del cuerpo. Los determinantes demostrativos indican distancia espacio-temporal. La derivación nominal se produce a través de la sufijación y, de manera muy productiva, a través de los compuestos nombre-adjetivo.

En cuanto a la morfología verbal, la lengua maká no expresa la categoría semántica de aspecto mediante una categoría morfológica verbal. Tampoco el tiempo es expresado en esta lengua morfológicamente. La temporalidad y el aspecto se infieren a partir de otros elementos de la oración como los adverbios o los determinantes demostrativos. Los verbos llevan prefijos personales de persona (1, 2, 3 y 4; esta última hace referencia a la primera persona plural inclusiva) y número (solo para la 4) que señalan, además, modo indicativo y no indicativo. Estos prefijos se dividen en seis clases según el grado de transitividad de la base. Sin embargo, no existe en maká una distinción tajante entre verbos transitivos e intransitivos, sino que la transitividad debe ser concebida en términos graduales más que dicotómicos ya que puede ser modificada mediante diferentes recursos morfosintácticos (Gerzenstein, 1991, p. 41 y ss., 1995).

Los verbos también pueden llevar sufijos locativos y direccionales. Estos sufijos pueden dividirse en dos grandes grupos: direccionales y aplicativos. Los primeros señalan la posición, locación o dirección del movimiento y rechazan la incorporación de un argumento objeto que dependa de ellos. Los aplicativos, por otra parte, permiten aumentar la valencia de la raíz verbal, ya que incorporan un argumento nominal objeto. Así, una raíz intransitiva más un aplicativo dará como resultado una construcción transitiva, mientras que una raíz transitiva acompañada de un aplicativo resultará en una construcción ditransitiva sin que por ello se modifique el índice pronominal de la raíz verbal. Además, estos aplicativos llevan prefijado un índice pronominal propio, que se ubica entre la raíz verbal y el aplicativo, y que se halla coindizado con el objeto aplicado (-yi-, -e-, -i/Ø-, -in- para la 1, 2, 3 y 4 persona respectivamente). Este índice es parcialmente idéntico a los prefijos personales de los verbos atributivos. Desde el punto de vista semántico, los argumentos incorporados por el aplicativo indican dirección, locación, beneficio o instrumento (Tacconi y Messineo en prensa; Tacconi, 2015).

Otra forma de aumentar la valencia verbal es la incorporación de sufijos causativos que se adjuntan tanto a los verbos transitivos como a los intransitivos. Estos sufijos pueden clasificarse en dos grandes grupos: los que expresan causación directa, formados con -t- (-t, -it/-hit, -ket/-kit, -inhet/-nhet), y aquellos que expresan causación indirecta, formados con -n- (-inen/-nen, -in/-hin, -henin/hinen). Estos sufijos permiten incorporar un argumento causado y alteran el índice pronominal de la raíz verbal (véase Tacconi 2016 para un análisis detallado de los causativos). La lengua también posee sufijos verbales desiderativos y reflexivos (Gerzenstein, 1995; Tacconi, 2015). 
No hay una clase definida de adjetivos, sin embargo, existen verbos atributivos que están a medio camino entre verbos y nombres, ya que reciben una marca de persona diferente a la del resto de los verbos y que es parcialmente idéntica a los prefijos posesivos de las personas del acto de habla (1 y 2). La lengua tampoco cuenta con una clase de adverbios de modo. Con respecto a la sintaxis, el orden preferido es VS para oraciones intransitivas y SVO para transitivas.

\subsection{Estudiar la diacronía desde la sincronía}

La teoría de la gramaticalización intenta explicar de qué manera se reestructuran las formas lingüísticas, en qué contextos específicos y en qué condiciones ocurren los cambios. Además, intenta determinar algunos factores prototípicos en este proceso, entre los cuales se encuentran el cambio semántico, el cambio estructural o formal, el reanálisis del significado y la coexistencia de varios estadios de gramaticalización en una etapa determinada de la lengua. Es decir, en el proceso de gramaticalización las formas son polisémicas (es el mismo morfema al que se le asignan diferentes significados y funciones).

Hay una oposición conceptual que atraviesa cualquier teoría sobre gramaticalización, y es la que se da entre formas independientes organizadas por reglas y principios (la gramática de la lengua) y formas fijas que pertenecen a un inventario (el léxico). Entre estos dos extremos se desarrolla un continuиm o escala que va desde las expresiones sintácticas relativamente libres a ítems léxicos. Esta oposición conceptual se plantea como una cuestión de composicionalidad en el sentido de que el significado de las expresiones sintácticas (en un extremo del continuum) puede ser interpretado a partir de la suma de sus componentes, mientras que el significado de los elementos léxicos (en el otro extremo) se vuelve opaco e institucionalizado.

En lenguas con una larga tradición de escritura, abordar estos aspectos implica comparar los registros de diversas épocas para analizar los diversos estadios de las construcciones gramaticalizadas. En lenguas de tradición oral, que solo han sido registradas por escrito durante el siglo XX, la tarea de registrar y analizar los procesos de gramaticalización no es imposible. Para ello utilizamos el método de reconstrucción interna, que parte de la premisa de que el cambio lingüístico deja ciertas señales en la estructura lingüística, que pueden observarse como irregularidades y que estas alternancias son evidencias de comportamientos diacrónicos anteriores (Fox, 1995, p. 145-147). Basándonos en este método, encaramos la tarea de registrar en la lengua maká algunas de las posibles instancias de gramaticalización. Los patrones de cambio o gramaticalización parecen seguir rutas universales, dado que se repiten en diversas lenguas del mundo que no están relacionadas genéticamente ni geográficamente. De esta forma, en una lengua como el maká con un registro acotado, podemos observar, a partir de los datos actuales, restos de estadios anteriores y confrontarlos con las mencionadas rutas universales para postular procesos de gramaticalización en curso. 
Presentamos aquí tres instancias de gramaticalización que se replican en otras lenguas. En primer lugar, lexemas que toman funciones de morfemas gramaticales. En estos casos, en un corte sincrónico pueden coexistir el lexema pleno y su forma homónima como morfema gramatical. En segundo lugar, morfemas con valores más concretos se gramaticalizan al tomar valores más abstractos o representar una nueva categoría gramatical. Por último, construcciones sintácticas pueden fusionarse y dar como resultado una construcción morfológica. Como detallamos en la introducción, abordamos distintas instancias de estos tres fenómenos en la lengua maká

En esta investigación utilizamos material recolectado en terreno personalmente desde el 2009 en adelante y lo contrastamos con fuentes de diversas épocas. Se utilizaron técnicas de elicitación, listas de palabras y oraciones, y traducción interlinear de narrativas. Todas las emisiones fueron grabadas y posteriormente transcriptas con la ayuda de los hablantes. Asimismo, se utilizaron fuentes de segunda mano, como vocabularios y apuntes gramaticales de los primeros estudiosos de la lengua (Belaieff, 1931, 1934, 1940; Schmidt, 1937), y el diccionario y el estudio descriptivo de Gerzenstein (1995, 1999) para registrar posibles cambios en la lengua.

\section{Rutas de gramaticalización: del léxico a la gramática y más allá}

Con respecto a los procesos de gramaticalización hemos podido identificar al menos cuatro ejemplos de este fenómeno en maká: el sufijo diminutivo proveniente del lexema -as 'hijo'; el surgimiento de una construcción causativa analítica; la refuncionalización de un sufijo desiderativo como marca aspectual y, finalmente, la aparición de sufijos aplicativos a partir de construcciones de verbos seriales. En los apartados siguientes analizamos en detalle cada una de estas instancias.

\subsection{Del lexema 'hijo' al diminutivo}

La morfología evaluativa es aquella que expresa la disminución, el aumento, el afecto o el desprecio mediante marcadores morfológicos específicos. Estos marcadores alteran la semántica de las bases a las que se adjuntan. En este sentido, el significado de los sufijos evaluativos es puramente atributivo y no referencial (Cúneo, 2014). Existen estudios que establecen una relación entre los términos de parentesco, específicamente de los términos para 'hijo/niño' y los marcadores de diminutivo (Heine y Kuteva, 2002; Grandi, 2011).

En maká la noción de diminutivo se expresa mediante el lexema $t$-as 'su hijo', con el marcador de tercera persona posesiva $t$ - cristalizado, que se adjunta a bases nominales. Esta forma constituye una palabra independiente que forma parte del vocabulario básico de la lengua y que además cumple, en un corte sincrónico, diversas funciones. Esta multifuncionalidad es la que nos permite postular un proceso de gramaticalización en curso, que detallamos a continuación. 
En primer lugar, tas 'su hijo' funciona como lexema pleno en tanto término de parentesco, como se observa en 1 .

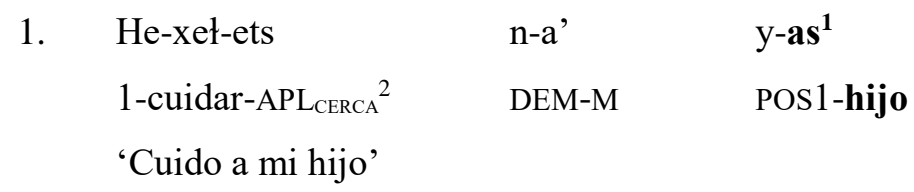

En segundo lugar, dentro del dominio etnobiológico, funciona como núcleo de compuestos que designan la cría de diversos animales:

2. a. tenuk

'gato'

b. tenuk-1-as

gato-POS3-hijo

'gatito' (lit. 'hijo del gato')

3. a. waka

'vaca'

b. waka-t-as

vaca-POS3-hijo

'ternero' (lit. 'hijo de la vaca')

4. a. kotseteyka

'cabra'

b. kotseteyka-t-as

cabra-POS3-hijo

'carnero' (lit. 'hijo de la cabra')

\footnotetext{
${ }^{1}$ Por pedido expreso del pueblo maká, que encuentra en la defensa de su alfabeto una forma más de preservación y mantenimiento de su lengua, y dada la facilidad de lectura para los hispanohablantes, hemos decidido utilizar el alfabeto estandarizado actual para transcribir los ejemplos. A continuación consignamos los grafemas y entre paréntesis el fonema que representan: a (/a/), e (/e/), f (/f/), h (/h/), i (/i/), j (/x/), k (/k/), l (/l/), m (/m/), n (/n/), o (/o/), p (/p/), q (/q/), s (/s/), t (/t/), ts $(/ \mathrm{ts} /),\left(\mathrm{u}(/ \mathrm{u} /), \mathrm{w}(/ \mathrm{w} /), \mathrm{y}(/ \mathrm{y} /), \mathrm{t}(/ \mathrm{t} /), \mathrm{x}(/ \chi /),{ }^{\prime}(/ \mathrm{r} /)\right.$.

${ }^{2}$ Las abreviaturas gramaticales utilizadas para las glosas son: A (sujeto activo); ADV (adverbio); APLABAJo (aplicativo abajo), APLCERCA (aplicativo cerca); APLDENTRo (aplicativo dentro); APLiNST (aplicativo instrumental); APLJunTo (aplicativo junto); APLMETA (aplicativo meta) ASP (aspecto); CAU(causativo); DEM (demostrativo); DES/ASP (desiderativo/aspectual); DIR (direccional); estar.PV (estar en posición vertical); F (femenino); M (masculino); NEG.IMP(negación de imperativo); NX (nexo); $\mathrm{O}_{\mathrm{APL}}\left(\right.$ índice $^{-}$ de Objeto aplicado); P (paciente); PL(plural); POS (marcador de poseedor); PRON (pronombre personal); PRSP (prospectivo); 1 (primera persona); 2 (segunda persona); 3 (tercera persona); 4 (primera persona plural inclusiva).
} 
En estos casos, el prefijo de poseedor de tercera persona está cristalizado y el compuesto es indivisible. Esta instancia configura entonces un primer paso en la instancia de gramaticalización ya que, si bien el segundo elemento del compuesto mantiene su contenido semántico básico 'hijo', se utiliza para designar a las crías de los animales.

En tercer lugar, en un paso más en la ruta de gramaticalización, se produce una extensión del significado básico y el lexema es utilizado como sufijo diminutivo. En este tercer uso, tas puede ser utilizado con objetos. Es decir, se produce un blanqueamiento de parte del significado debido a que no implica una relación de parentesco, sino una diferencia de tamaños:

5. a. phof

'pelota'

b. phof-t-as-i

pelota-POS3-hijo-F

'pelotita' (lit. 'hija de la pelota')

6. a. tinaj

'bolsa'

b. tinaj-t-as

bolsa-POS3-hijo

'bolsito'

En algunos casos, el diminutivo se utiliza para señalar una variante del elemento y no necesariamente un objeto más chico:

7. a. koyoyoy

'carro'

b. koyoyoy-t-as-i

carro-POS3-hijo-F

'carretilla'

8. a. niyak

'soga'

b. niyak-t-as

soga-POS3-hijo

'piolín' 
Finalmente, en un paso más en la cadena de gramaticalización, el sufijo diminutivo es utilizado como sufijo afectivo. Como observamos en los ejemplos 9 a 11 donde el lexema resultante no se refiere literalmente al hijo de mi abuelo/tío/nieto, sino que se utiliza como un vocativo que expresa cariño o afecto.

9. a. y-ewket

POS1-abuelo

'mi abuelo'

b. y-ewket-t-as

POS1-abuelo-POS3-hijo

'mi abuelito' (lit. “el hijo de mi abuelo')

10. a. totok $^{3}$

'mi tío'

b. totok-t-as

mi.tío-POS3-hijo

'mi tí́to' (lit. 'el hijo de mi tío')

11. a. y-uk

POS1-nieto

'mi nieto'

b. $\mathbf{y}$-uk-t-as

POS1-nieto-POS3-hijo

'mi nietito'

Es decir que para este sufijo particular la ruta de gramaticalización ha pasado por tres estadios: lexema independiente, constituyente de un compuesto y, finalmente, sufijo gramatical. Es importante resaltar que, en este caso, todos los estadios de la ruta de gramaticalización coexisten en un corte sincrónico de la lengua. Probablemente, esto se deba a que tas 'su hijo' pertenece al llamado vocabulario básico de la lengua y por esto es difícil que desaparezca su uso como lexema independiente.

\footnotetext{
${ }^{3}$ Este lexema no sigue el paradigma posesivo en la primera persona.
} 


\subsection{Del verbo 'decir’ a la construcción causativa analítica}

La causatividad puede expresarse de tres maneras: léxica, morfológica y analítica. En muchos casos, el origen de las construcciones causativas morfológicas se encuentra en estructuras analíticas o en diversos lexemas de la lengua que se gramaticalizan. Un caso frecuente en las lenguas del mundo es la gramaticalización del verbo 'decir' que se cristaliza en una construcción causativa; esto sucede, por ejemplo, en lenguas como el baka (familia ubangi, Níger-Congo) y el lezgiano (caucásica septentrional), entre otras, de acuerdo con Heine y Kuteva (2002, p. 261).

Como mencionamos en la introducción, el maká expresa la causatividad a través de sufijos verbales que incorporan un participante al evento básico que funciona como hostigador de la acción más o menos directamente. No obstante, se ha registrado también en maká una construcción causativa analítica que es menos productiva y que presenta indicios de gramaticalización. Esta construcción se forma con el verbo -it'ij 'decir' más un verbo impersonal, un verbo atributivo o un nombre.

En dichas construcciones, el verbo -it' $i j$ funciona como un auxiliar que lleva las marcas de persona y modo, mientras que el segundo elemento aporta la información semántica del evento que se lleva a cabo, pero no lleva índices pronominales. De acuerdo con la tipología de Anderson (2006), la construcción de verbo auxiliar del maká sería una estructura con el auxiliar como núcleo flexivo (auxiliary headed) dado que, si bien el núcleo semántico lo constituye el verbo principal, el núcleo flexivo, es decir aquel que lleva las marcas de flexión, es el auxiliar.

En los ejemplos que se muestran a continuación consignamos las diversas funciones del verbo -it'ij 'decir' en la lengua maká. En 12 se observa su utilización como verbo pleno con el valor semántico típico de los verbos de comunicación en el discurso directo:

\begin{tabular}{|c|c|c|c|c|c|}
\hline 12. “¡Hasu’uj & i-wejint & $1+i-i-j$ & $n-a^{\prime}$ & tenuk & qu' \\
\hline NEG.IMP & 2-dejar- & $r-\mathrm{O}_{\mathrm{APL}} 3-\mathrm{APL}_{\mathrm{INST}}$ & DEM-M & gato & $\mathrm{NX}$ \\
\hline ni-wum-ju' & & $n-a^{\prime}$ & ofotax!" & & \\
\hline 3-atacar-DIR & & DEM-M & paloma & & \\
\hline$y$-it'ij & $\mathrm{p}-\mathrm{a}^{\prime}$ & Gilbe & & & \\
\hline 3-decir & DEM-M & Gilbe & & & \\
\hline
\end{tabular}

En 13 es utilizado como verbo de comunicación en el discurso indirecto, por lo que toma una oración subordinada mediante el nexo 'qu'. En este contexto, es frecuente su utilización con el valor de orden o mandato: 


\begin{tabular}{|c|c|c|c|c|}
\hline 13. n-a' & jukhew & $y$-it'ij-Ø-ets ${ }^{4}$ & k-e' & qu' \\
\hline DEM-M & hombre & 3-decir- $\mathrm{O}_{\mathrm{APL}} 3-\mathrm{APL}_{\mathrm{CERCA}}$ & DEM-F mujer & $\mathrm{NX}$ \\
\hline
\end{tabular}

'El hombre le dice a su mujer que cocine'

Finalmente, el verbo -it'ij puede ocurrir en una construcción causativa analítica. En los ejemplos 14 a 17a observamos el verbo utilizado en una construcción no causativa, mientras que en b mostramos el uso de los mismos verbos con la construcción causativa. Es altamente probable que esta última construcción tenga su origen en la utilización del verbo decir como verbo de mandato u orden. En cuanto a la estructura, el verbo auxiliar lleva los prefijos personales, mientras que el verbo auxiliar aporta la información semántica. Este segundo verbo puede ser un verbo transitivo típico, un verbo impersonal qhof 'abrir' o un verbo atributivo.
14. a. h-ew-q'ul-ju'
n-a'
najak
1-?-doblar-DIR
DEM-M
palo

'Arqueo un palo'

b. h-it'ij

1-decir n-e'

DEM-F y-aqhuts

POS1-rodilla

'Flexiono mi rodilla' (Lit. 'Digo doblar mi rodilla, no utilizo mis manos')

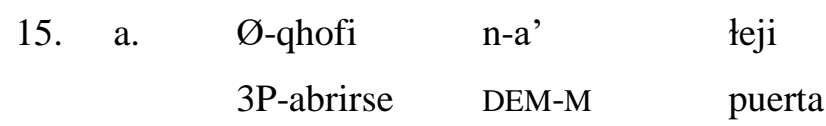

'La puerta se abrió'

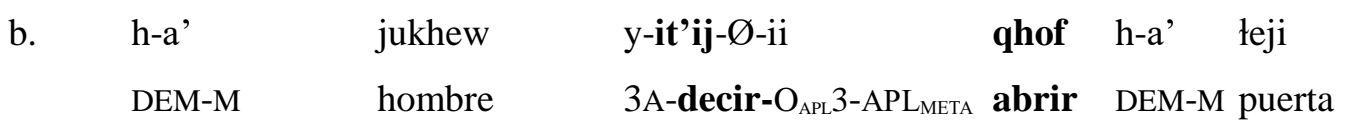

'El hombre abrió la puerta'

16. a. Ø-s'e' n-a' junwat

3P-ser.colorado DEM-M pájaro

'El pájaro es colorado'

b. h-it'ij s'e'

1A-decir ser.colorado

'Coloreo' (lit. 'Digo ser colorado')

\footnotetext{
${ }^{4}$ El aplicativo -ets transitiviza el verbo decir y permite incorporar el argumento efu 'mujer'.
} 


$$
\begin{aligned}
& \text { 17. n-e' junatai y-it'ij k'aj n-e' tapas-its } \\
& \text { DEM-F pájaros 3A-decir abrir DEM-PL pico-PL }
\end{aligned}
$$

'Los pájaros abren sus picos' (Lit. 'Los pájaros hacen abrir sus picos')

Un caso atípico, del que hemos registrado una sola estructura, es el del verbo -it'ij acompañado por un sustantivo como en el ejemplo 18 con petetpetet 'taladro':

\begin{tabular}{|c|c|c|c|c|}
\hline K-e' & efu & $y-\mathbf{i t}^{\prime} \mathbf{i j}$ & petetpetet & h-a' \\
\hline DEM-F & mujer & 3A-decir & taladro & DEM-M \\
\hline
\end{tabular}

$\begin{array}{lllll}\text { 18. a. } & \text { H-a' } & \text { omełas } & \text { y-it'ij } & \text { petetpetet } \\ & \text { DEM-M } & \text { niño } & \text { 3A-decir } & \text { taladro } \\ & \text { 'El niño da vueltas' } & & \end{array}$

'La mujer hace que el niño dé vueltas'

Los ejemplos previos dan cuenta de que la construcción causativa analítica se halla en maká en un proceso de gramaticalización, en el que el verbo -it'ij pierde su significado léxico original para actuar como un verbo auxiliar con valor causativo.

Como mencionamos al comienzo de este apartado, la gramaticalización del verbo 'decir' con valor causal ha sido documentada en otras lenguas del mundo como un proceso que selecciona una característica semántica sobresaliente de un verbo y da lugar a un marcador gramatical. En el caso de lenguas de la región chaqueña, González (2015, p. 161-163) la registra para el toba del este de Formosa (familia guaycurú). Sin embargo, a diferencia de lo que sucede en el maká, el verbo 'decir' del toba lleva la marca impersonal, mientras que el verbo principal aparece flexionado. Según la tipología de Anderson (2006) podríamos clasificarlo de núcleo léxico (lexical headed), como se muestra en el ejemplo 19:

19. d-ashiw-aGan na qo-yin

3I-fumar-AGT DAC P.IMP-3I-decir

'Le dicen que fume / Lo hacen fumar'

\subsection{Del desiderativo al aspecto ingresivo}

Una tercera instancia de gramaticalización frecuente en las lenguas del mundo es aquella que tiene su origen en marcadores o lexemas desiderativos que evolucionan en marcadores de aspecto. Esta ruta puede tener su origen en lexemas como 'querer', 'desear' o 'amar' que pueden desarrollarse 
diacrónicamente como marcadores de futuro o aspecto inminente. De acuerdo con la clasificación de Heine y Kuteva (2002, p. 311) Querer > marcador ingresivo 'estar por' o Amar > marcador ingresivo5.

En el caso del maká, la construcción desiderativa puede expresarse a través del verbo su'un 'amar/desear', ejemplo 20, que señala alguien o algo que el sujeto quiere; o mediante el sufijo desiderativo -hiyu' y sus alomorfos (-i/uyu', -yu', $-u^{\prime}$ ), este conjunto de sufijos indican deseo o voluntad de realizar la acción que denota el verbo al que se sufija, ejemplos 21 a 23. La incorporación de este sufijo provoca el cambio de los índices pronominales del verbo base y toma los de la conjugación 3 que indican un sujeto paciente:

20
hi-su'un
n-a'
li-wihitjii
ts-e' Andrea
1-querer
DEM-M
POS3-camisa
DEM-F Andrea

'Quiero la camisa de Andrea'

21. n-otoy-hiyu'

$$
\begin{array}{ll}
\text { ts-a' } & \text { Andrés } \\
\text { DEM-M } & \text { Andrés }
\end{array}
$$

3-bailar-DES

'Andrés quiere bailar'

\begin{tabular}{|c|c|c|}
\hline yakha’ & tsi-n-hiyu-pji & $n-a^{\prime}$ \\
\hline PRON1 & 3-estar.PV-DES-APL $L_{\mathrm{LOC}}$ & DEM-M \\
\hline
\end{tabular}

22.

$\begin{array}{llll}\text { hats } & \text { n-ek-uyu' } & \text { ts-a' } & \text { Juan } \\ \text { ADV } & \text { 3-comer-DES } & \text { DEM-M } & \text { Juan }\end{array}$

'Juan ya quiere comer'

'Yo quiero subir al caballo'

Si bien, como señalamos en la introducción, el maká no posee marcadores aspectuales obligatorios, este grupo de sufijos parece haber extendido sus funciones, puesto que además del valor desiderativo cumple la función de sufijo aspectual y señala acciones inminentes.

24.
n-ots-hiyu-ju'
n-a'
omelhas
3-caminar-DES/ASP-DIR
DEM-M
niño
'El niño quiere caminar/está por caminar'

\footnotetext{
${ }^{5}$ En el original "WANT > proximative marker "be about to"” / "LOVE > proximative marker".
} 
Aunque en el ejemplo precedente la interpretación puede ser ambigua, dado que podría indicar que el niño desea caminar tanto como que está por caminar, en 25 podemos observar que claramente el sufijo -iyu ha perdido su valor volitivo, ya que puede adjuntarse a verbos cuyos sujetos, por ser inanimados (como la 'olla'), no pueden tener volición.

25.

$$
\begin{array}{lll}
\text { n-am-iyu-ju' } & \text { n-a' } & \text { atxat } \\
\text { 3-llegar-DES/ASP-DIR } & \text { DEM-M } & \text { olla }
\end{array}
$$

'La olla está por caerse'

Además, en maká, este sufijo con valor aspectual puede combinarse con el sufijo evaluativo tax para indicar aspecto incompletivo, 'acción realizada en vano', como observamos a continuación:

$$
\begin{aligned}
& \text { 26. n-ekum-hiyu-tax-i p-e' yi-wen (qayiwumpa' t'uniki) } \\
& \text { 3-tocar-DES-ASP-APL LOC DEM-F 3-ver }
\end{aligned}
$$

El sufijo derivativo evaluativo -tax normalmente crea nombres que designan variedades anómalas o no autóctonas de animales o plantas (cfr. Messineo y Tacconi, 2010). Podemos pensar que la conexión entre ambos sufijos se produce porque en su función verbal señala aquello que se 'frustra' o 'no es'; y en su uso nominal señala la variedad de una especie, la que 'no es'. Es posible, entonces, establecer una relación semántica entre ambos significados.

La doble función del sufijo -hiyu' (y sus alomorfos) como desiderativo y como aspectual ocurre también en las lenguas mataguayas, como el chorote. Carol (2014, p. 191) documenta el sufijo jayil-jayu:

27.
a. a-lakyen
$1 \mathrm{~S}_{\mathrm{A}-\mathrm{jugar}}$
'Juego'
b. si-lakyejnayi'
1-jugar+PRSP
'Voy a jugar/quiero jugar'

Construcciones similares han sido registradas por Fabre (2014) para el nivaclé, y es común también en otras lenguas de la región, como registran Messineo y Cúneo (2010, p. 243) para el toba con una construcción de verbos seriales. 


\subsection{De verbos seriales a construcciones aplicativas locativas}

El desarrollo de sufijos locativos y direccionales a partir de procesos de gramaticalización ha sido señalado en varias lenguas del mundo. En el caso de los aplicativos, es frecuente que estos provengan de la cristalización de compuestos verbo-verbo o construcciones de verbos seriales; en otros casos, de frases verbo-adposición (cfr. entre otros Mithun, 2002; Heine y Kuteva, 2002 para un detalle de los diversos orígenes de los aplicativos). Esto explica, según Mithun (2002, p. 97), que el verbo principal de la construcción gramaticalizada tome como argumentos el sujeto gramatical de la raíz verbal y el objeto de la adposición o del segundo verbo gramaticalizado como aplicativo. Empero, la construcción resultante no es equivalente a su par histórico ni en sus propiedades formales ni en las funcionales.

De igual manera, los aplicativos en maká parecen también tener su origen en construcciones de verbos seriales de un estadio anterior de la lengua. La evidencia de este fenómeno no surge en este caso de la multifuncionalidad sincrónica de los aplicativos, sino de dos fuentes principales: en primer lugar, postulamos que los índices pronominales prefijados al aplicativo constituyen el resto fónico de los índices pronominales del segundo verbo de la construcción, como se muestra en los ejemplos que analizamos a continuación; en segundo lugar, los datos provenientes de otras lenguas de la familia en las que sí existe la multifuncionalidad de los aplicativos apoyan nuestra hipótesis. A continuación desarrollamos ambas evidencias.

Como señalamos en la introducción, la lengua cuenta con un conjunto de sufijos aplicativos que incorporan un argumento objeto que semánticamente expresa contenido locativo, direccional, instrumental o benefactivo. Estos aplicativos se adjuntan tanto a verbos transitivos como intransitivos. En el caso de los locativos y direccionales, que especifican la locación y la trayectoria del movimiento denotado, hipotetizamos que su origen puede hallarse en antiguas construcciones de verbos seriales como las que se reconstruyen a continuación. En estas el segundo verbo de la serie sería un verbo estativo o locativo básico:

28.

\begin{tabular}{|c|c|}
\hline ts-am-i-fi & $n-e^{\prime}$ \\
\hline 1P-llegar-O ${ }_{\mathrm{APL}} 3-\mathrm{APL}_{\mathrm{ABAJO}}$ & DEM-F \\
\hline
\end{tabular}

'Me caí al pie del árbol.'

Reconstrucción hipotética

$\begin{array}{lllll}* \text { ts-am } & + & \text { i-fi } & \text { n-e' } & \text { najkak } \\ \text { 1P-caer } & + & \text { 3P-estar.debajo } & \text { DEM-F } & \text { árbol } \\ \text { 'Yo me caí' } & + & \text { 'Está al pie el árbol' } & & \end{array}$

29. he-npuli- $\varnothing$-ji' $\quad$ n-a' haqqi' 
1-bañarse-O-O ${ }_{\mathrm{APL}}$ 3-APL ${ }_{\mathrm{DENTRO}} \quad$ DEM-M

'Me baño en el río'

Reconstrucción hipotética

$\begin{array}{lllll}\text { *he-npulij } & + & \text { i-ji } & \text { n-a' } & \text { haqqi’ } \\ \text { 1-bañar } & + & \text { 3P-estar.dentro } & \text { DEM-M } & \text { río }\end{array}$

'Me baño' + + + Es tridimensional el río' ('El río es mi recipiente')

30. $\quad y$-at'in-i-fi n-a' wits'ojilax n-a' wit'itila

3-esconder-O APL $_{\text {3-APL }}$ ABAJO DEM-M silla DEM-M collar

'Escondió el collar debajo de la silla'

Reconstrucción hipotética

$\begin{array}{lllll}* \begin{array}{lll}* \text {-at'in } \\ \text { n-esconder }\end{array} \text { DEM-M collar } & + & \boldsymbol{i} \text { - }(V) f i & \text { n-a' } & \text { wits'ojilax } \\ \text { 3-itila } & + & \text { 3P-estar.debajo } & \text { DEM-M silla } \\ \text { 'Él esconde al collar' } & + & \text { 'Está al pie la silla', } & \end{array}$

31. Ø-ts'ap'a-in-jup

3-estar.parado-O-O ${ }_{\text {APL }}$-APLJUNTO

'Él/ Ella está parado al lado de nosotros'

Reconstrucción hipotética

$\begin{array}{lll}\text { * Ø-ts'ap'a } & + & \text { in- }(V) \text { jup } \\ \text { 3-estar.parado } & + & \mathbf{4 P - e s t a r . a l . l a d o} \\ \text { 'Él está parado' } & + & \text { 'Estamos al lado' }\end{array}$

Como se puede observar en los ejemplos 28 a 31, el aplicativo lleva prefijado una marca de persona que se coindiza con el objeto aplicado. De acuerdo con nuestra hipótesis, ese índice sería el resto fónico del índice pronominal de sujeto del segundo verbo de la serie. Es probable que, a lo largo del proceso de gramaticalización, este índice haya pasado de indexar el sujeto de un verbo atributivo locativo (segundos ejemplos de la serie 28 a 31) a indexar el objeto aplicado.

Esta hipótesis es respaldada por datos como los de Bakker (2013), quien señala el origen verbal de las adposiciones en diversas lenguas. El autor indica que es frecuente en las lenguas del mundo que los clíticos o las adposiciones lleven marcador de persona. La forma fonológica de este marcador puede ser un indicio de que estos marcadores ya estaban presentes en el precursor verbal de la adposición o clítico. En la lengua maká se cumple la última afirmación, la forma fonológica del objeto aplicado es parcialmente semejante a la de los prefijos personales de los verbos atributivos, pues ambos requieren un sujeto paciente. Es decir, que el marcador de objeto (Paciente) de la 
construcción actual sería el resto de una marca de participante pacientivo coincidente con la de los predicados atributivos. Esto es coincidente también con el orden VS esperado en las oraciones intransitivas. Aun cuando suponer que el índice de objeto del aplicativo es un resto de una marca de sujeto pacientivo en la reconstrucción puede resultar arriesgado, consideramos que las evidencias provenientes de lenguas de la misma familia, que presentamos a continuación, constituyen al menos un indicio de que este sería el caso ${ }^{6}$.

Si bien en una muestra sincrónica de la lengua no encontramos construcciones de verbos seriales que involucren verbos locativos o de movimiento, la comparación con otras lenguas de la familia parece confirmar nuestra hipótesis. El wichí, por ejemplo, parece estar un paso atrás en la cadena de gramaticalización, debido a que, como señala Nercesian (2014, p. 255), coexisten las construcciones de verbos seriales (verbo general más verbo de movimiento) y la construcción de verbo más aplicativo. La autora distingue dos clases de aplicativos según el grado de gramaticalización que presenten. Los de la Clase I, que no se presentan como formas libres y están sincrónicamente colexicalizados con bases verbales y los de la Clase II que coexisten con la forma libre (un verbo) en sincronía (2014, p. 250). Transcribimos aquí ejemplos de la Clase II con el mismo morfema en sus dos funciones, verbo libre en una construcción serial, ejemplo 32; y aplicativo, ejemplo 33:

$\begin{array}{lll}\text { 32. } & \text { n'-nay } & \text { n'-ihi } \\ \text { 1-bañar } & \text { 1-estar.en } \\ \text { 'me baño en el río' (lit. 'n } & \\ \text { 33. } & \text { n'-nay-hi } & \text { tewukw } \\ \text { 1-bañar-APL } & \text { río } \\ & \text { 'me baño en el río' }\end{array}$

La autora interpreta estos datos como indicio del origen de los aplicativos en construcciones de verbos seriales, dado que los verbos de posición y movimiento se ven involucrados en construcciones de verbos seriales con una función similar a la del aplicativo, la de agregar un argumento más a la construcción con un rol semántico específico (Nercesian, 2014, p. 255). Confróntense los ejemplos 32 y 33 del wichí con el 29 de la lengua maká, en los que el aplicativo es el mismo (-hi o -ji según la transcripción). Proponemos que en el caso del maká, el índice que acompaña al aplicativo actualmente puede haber codificado antiguamente al sujeto de la oración, de ahí las traducciones propuestas. Dicho sujeto codificaba el objeto o la persona respecto al cual se orientaba la acción.

\footnotetext{
${ }^{6}$ Agradezco las acertadas sugerencias del evaluador anónimo con respecto a las contrucciones con aplicativos.

${ }^{7}$ La glosa es nuestra.
} 
De esta forma, podemos postular para el maká un estadio más avanzado en la ruta de gramaticalización en el que el segundo verbo de la serie ha sido completamente absorbido por el verbo principal y funciona como aplicativo.

\section{Conclusiones}

A lo largo de este artículo hemos abordado diversas rutas de gramaticalización en la lengua maká: la formación del diminutivo y el afectivo a partir del lexema tas 'su hijo'; el surgimiento de una construcción causativa analítica con el verbo -it'ij 'decir'; la incorporación de funciones más gramaticales con valores aspectuales del sufijo desiderativo -iyu y sus alomorfos; y, finalmente, el hipotético origen de los aplicativos en construcciones de verbos seriales. A partir de los ejemplos analizados podemos concluir que diversos ítems morfológicos observables en la sincronía de la lengua maká se corresponden con estructuras sintácticas o menos gramaticales en la diacronía.

Todas estas instancias de análisis contribuyen a sostener que es posible el estudio de procesos de gramaticalización aun en lenguas con escaso registro histórico. Si bien no es viable encontrar todas las características o pasos de la gramaticalización, con un relevamiento sincrónico se puede hipotetizar el origen de diversas construcciones. Asimismo, la comparación con otras lenguas de la familia, del área y del mundo arroja evidencia que, acompañada con el análisis inmanente de los datos, permite recomponer procesos de gramaticalización cuyos rastros permanecen ocultos en el análisis estrictamente sincrónico de una lengua.

\section{Referencias bibliográficas}

Anderson, Gregory. (2006). Auxiliary verb constructions. Oxford: Oxford University Press.

Bakker, Dik. (2013). "Person Marking on Adpositions”. En: Dryer \& Haspelmath (eds.): Cap. 48.

Belaieff, Juan. 1931. "El vocabulario Maccá”. Revista de la Sociedad Científica del Paraguay, III (2), 53-67.

Belaieff, Juan. (1934). “El vocabulario maccá. Claves y apuntes gramaticales”. Revista de la Sociedad Científica del Paraguay, III (4), 124-30.

Belaieff, Juan. (1940). "El Maccá”. En: Revista de la Sociedad Científica del Paraguay, IV(6).

Carol, Javier. 2014. Lengua chorote (mataguayo). Estudio fonológico y morfosintáctico. Munich: LINCOM Studies in Native American Linguistics.

Cúneo, Paola. (2014). “Augmentative in Toba (Guaycuruan): Form and Function”. En: Danielsen Swintha, Hanss, Katja y Zúñiga Fernando (eds.), 51-77.

Danielsen, Swintha et al. (eds.). (2014). Word formation in South American Languages. Berlin: John Benjamins Publising Company. 
DGEEC. (2014). Pueblos Indígenas en el Paraguay. Resultados finales de población y viviendas 2012. Fernando de la Mora: Dirección General de Estadísticas, Encuestas y Censos.

Dryer, Matthew \& Martin Haspelmath (eds.) The World Atlas of Language Structures Online. Leipzig: Max Planck Institute for Evolutionary Anthropology. (Available online at http://wals.info/chapter/48, Accessed on 2018-01-17).

Fabre, Alain. (2014). Estudio gramatical de la lengua nivaclé. Recuperado de http://www.etnolinguistica.org/biblio:fabre-2014-estudio.

Fox, Anthony. (1995). Linguistic Reconstruction. An Introduction to theory and method. Oxford: Oxford University Press.

Gerzenstein, Ana. (1991). Grados de transitividad en el verbo maká. En: Gerzenstein (Coord.) Temas de lingüística aborigen. Buenos Aires: UBA, FFYL, 39-56.

Gerzenstein, Ana. (1995). El maká. Estudio descriptivo. Buenos Aires: Facultad de Filosofía y Letras, Universidad de Buenos Aires.

Gerzenstein, Ana. (1999). Diccionario Etnolingüístico Maká-Español (DELME). Buenos Aires: Instituto de Lingüística, Facultad de Filosofía y Letras, Universidad de Buenos Aires.

González, Raúl. (2015). Estudio fonológico y morfosintáctico de la lengua toba hablada en el este de la provincia de Formosa (Argentina). München: LINCOM Studies in Native American Linguistics.

Grandi, Nicola. (2011). "Renewal and Innovation in the Emergence of Indo-European Evaluative Morphology”. En: Körtvélyessy y Stekauer (Eds.) Diminutives and Augmentatives in the Languages of the World, Lexis: e-journal in English lexicology, 6, 5-25. Recuperado de http://lexis.univ-lyon3.fr/IMG/pdf/Lexis_6.pdf

Heine, Bernd y Tania Kuteva. (2002). World Lexicon of Grammaticalization. Cambridge: Cambridge University Press.

Hopper, Paul y Elizabeth Traugott. (1993). Grammaticalization. Cambridge: Cambridge University Press.

Lehmann, Christian. (2004). "Theory and method in grammaticalization". Zeitschrift für Germanistische Linguistik, 32 (2), 152-187.

Messineo, Cristina et al. (comps.). (2010). Léxico y categorización etnobiológica en grupos indígenas del Gran Chaco Santa Rosa: Editorial de la Universidad Nacional de La Pampa (EDUNLAPam).

Messineo, Cristina y Paola Cúneo. (2009-2010). "Construcciones seriales en toba (guaycurú)". Amerindia, 33 (34), 217-248.

Messineo, Cristina y Temis Tacconi. (2010). "Recursos de formación del léxico en maká (mataguayo): zoonimia y fitonimia”. En: Messineo, Scarpa y Tola (comps.) Léxico y categorización etnobiológica en grupos indígenas del Gran Chaco, 83-116. 
Mithun, Marianne. (2002). "Understanding and explaining applicatives". En: Andronis, Ball, Elston y Neuvel (Eds.) Proceedings of the Thirty-seventh Meeting of the Chicago Linguistic Society: Functionalism and formalism in Linguistic Theory. Chicago, 73-98.

Nercesian, Verónica. (2014). Wichi lhomtes. Estudio de la gramática y la interacción fonologíamorfología-sintaxis-semántica. München: LINCOM

Pueblo Maká. (s/f). Hisu'unji qu' Hiyinenik'i'. Mi primer libro de lectura Maká. Asunción: Dirección General de Educación Escolar Indígena.

Schmidt, Max. (1937). "Vocabulario de la lengua maká". Revista de la Sociedad Científica del Paraguay, 4 (2), 68-85.

Tacconi Temis y Cristina Messineo. En prensa. "Construcciones aplicativas en maká: morfosintaxis y semántica”. En: Guerrero (Ed.) Adposiciones y elementos de su tipo en lenguas amerindias. México: UNAM.

Tacconi Temis. (2015). Procesos de formación de palabras en maká (mataguayo). Tesis doctoral inédita. Universidad de Buenos Aires.

Tacconi Temis. (2016). “Construcciones causativas y desiderativas en maká (mataguayo)”. LIAMES $16,95-117$.

\section{@ $\odot \Theta$}

Esta obra está bajo una licencia de Creative Commons Reconocimiento-NoComercial-SinObraDerivada

4.0 Internacional 